\title{
Neurocognitive Basis of Schizophrenia: Information Processing Abnormalities and Clues for Treatment
}

\author{
André Aleman ${ }^{1,2}$ \\ ${ }^{1}$ Department of Neuroscience, University Medical Center Groningen, University of Groningen, 9700 RB Groningen, The Netherlands \\ ${ }^{2}$ Department of Psychology, University of Groningen, 9712 CP Groningen, The Netherlands
}

Correspondence should be addressed to André Aleman; a.aleman@med.umcg.nl

Received 10 September 2013; Accepted 8 December 2013; Published 9 February 2014

Academic Editor: Daniela Schulz

Copyright (C) 2014 André Aleman. This is an open access article distributed under the Creative Commons Attribution License, which permits unrestricted use, distribution, and reproduction in any medium, provided the original work is properly cited.

\begin{abstract}
Schizophrenia is a chronic and severe psychiatric disorder that affects all aspects of patients' lives. Over the past decades, research applying methods from psychology and neuroscience has increasingly been zooming in on specific information processing abnormalities in schizophrenia. Impaired activation of and connectivity between frontotemporal, frontoparietal, and frontostriatal brain networks subserving cognitive functioning and integration of cognition and emotion has been consistently reported. Major issues in schizophrenia research concern the cognitive and neural basis of hallucinations, abnormalities in cognitive-emotional processing, social cognition (including theory of mind), poor awareness of illness, and apathy. Recent findings from cognitive neuroscience studies in these areas are discussed. The findings may have implications for treatment, for example, noninvasive neurostimulation of specific brain areas. Ultimately, a better understanding of the cognitive neuroscience of schizophrenia will pave the way for the development of effective treatment strategies.
\end{abstract}

\section{Introduction: Schizophrenia}

Schizophrenia belongs to the most severe psychiatric disorders. It is characterized by hallucinations, delusions, cognitive impairment, and social withdrawal [1]. In most cases, people with this diagnosis might have difficulty to think clearly, to distinguish reality from fantasy, to react in an emotionally appropriate way, and to interact with others. One of the most tragic features of the disorder is the early appearance of the symptoms, usually between ages 20 and 35, with devastating effects on social relationships, education, and starting a professional career, thereby ruining all promises of early adulthood. Approximately $15 \%$ of patients with schizophrenia commit suicide. The general population lifetime prevalence of schizophrenia is approximately $1.0 \%$, and the annual incidence rate is between 0.16 and $0.42 / 1000$ persons at risk [2]. Although textbooks used to state that schizophrenia affects men and women equally (e.g., Kaplan et al. [3]), evidence is accumulating that men are at higher risk for schizophrenia [4], especially during adolescence and young adulthood and especially for schizophrenia with negative symptoms.

Symptoms of schizophrenia include delusions, hallucinations, disorganized speech, grossly disorganized or catatonic behavior, affective flattening, alogia, and avolition [5]. None of these symptoms is pathognomonic for schizophrenia [6]. Symptoms of schizophrenia have been characterized to be "waxing and waning", referring to the acute and chronic phases in which symptoms can be present. Acute symptoms are usually so-called "positive" symptoms (which are present in schizophrenia but not in healthy individuals), such as delusions and hallucinations. On the other hand, negative symptoms (the absence of functions that are present in healthy individuals), such as affective flattening and avolition, are more persistent and have stronger prognostic importance $[7,8]$.

\section{Cognitive Dysfunction}

It is surprising that cognitive dysfunction, although it is widely recognized to be a hallmark of schizophrenia, is not listed among the symptoms of schizophrenia in the American Psychiatric Association's Diagnostic and Statistical Manual, be it version DSM-IV or the new DSM 5. Indeed, numerous neuropsychological investigations have established that patients with schizophrenia suffer from significant deficits 
in attention, memory, executive functioning, and general intellectual abilities $[9,10]$. Large and stable effect sizes have been reported for memory impairment [11]. In a recent metaanalysis, Reichenberg and Harvey [10] presented a quantitative integration of the published literature showing that the most severe impairments are apparent in episodic memory and executive control processes (with effect sizes around 1SD in magnitude), evident against a background of a generalized cognitive deficit. This was recently confirmed in yet another meta-analysis that concluded that there is a large and stable, general cognitive deficit that is similar across the world and has not changed over the past decades, despite some changes in diagnostic criteria [12]. The neuropsychological impairments potentially represent genetic liability to the disorder: similar, yet milder, impairments are evident in schizophrenia patients even before the onset of psychotic symptoms, as well as in the nonpsychotic relatives of schizophrenia patients. In relatives, meta-analyses have established effect sizes of medium magnitude (around 0.5 SD), with strongest impairment in executive functioning and memory $[13,14]$. However, this effect size also implies that a considerable proportion of relatives have no neuropsychological impairment.

Another meta-analysis of studies conducted with patients identified processing speed inefficiency as the largest single cognitive impairment in schizophrenia [15]. However, in a meta-analysis of moderator variables affecting processing speed in patients with schizophrenia, Knowles et al. [16] showed that the processing speed impairment is substantially affected by several moderating factors, in particular antipsychotic medication dosage.

It should be noted that studies of cognitive impairment in schizophrenia have used a large number of different neuropsychological tests. Such heterogeneity of measurement poses problems for interpretation as comparison between different studies is difficult when the measures have different characteristics (e.g., slight differences in cognitive processes that are targeted, but also differences in duration of the test, reliability, validity, etc.). Therefore, the consensus battery MATRICS [17] that was proposed by a team of investigators coordinated by the National Institutes of Mental Health (NIMH) is a laudable development that can improve comparability which is especially needed for treatment studies in which the effect of an intervention (be it pharmacotherapy or psychotherapy) is assessed on cognitive functioning. MATRICS is an abbreviation of "Measurement and Treatment Research to Improve Cognition in Schizophrenia." The consensus cognitive battery includes tests with adequate reliability and validity in the following domains: speed of processing, attention/vigilance, working memory, verbal learning and memory, visual learning and memory, reasoning and problem solving, and social cognition (see http://www.matricsinc.org/MCCB.htm).

Another important point to be aware of concerns the heterogeneity of the cognitive impairment itself among patients. That is, although on a group level patients consistently perform worse than healthy control subjects, this is not necessarily the case at an individual level. Thus, a small subgroup of patients is neuropsychologically normal; that is, their performance on neuropsychological tests of attention, memory and executive functioning is in the normal range of age- and education-matched samples [18]. However, a study by Wilk et al. [19] revealed that, even after matching schizophrenia patients to control subjects on IQ, patients still exhibited performance deficits in memory and speed visual processing.

With regard to brain regions underlying these deficits in cognitive functioning, it is now clear that frontal and temporal cortices are strongly involved. For example, the classic finding by Weinberger et al. [20] of reduced DLPFC activation has been widely replicated [21]. On the other hand several studies have reported increased prefrontal activation during working memory tasks $[22,23]$. However, this may be observed primarily in tasks that are difficult for patients, but which they are still able to perform. When the task becomes too difficult and their performance is worse than that of comparison subjects, patients will typically show less activation. With regard to the temporal cortex, its involvement in functional scans during episodic memory has also consistently been reported with compromised activation in schizophrenia [24].

Even though the importance of the frontal and temporal cortex is well recognized, compromised frontostriatal and frontoparietal function is also well established in schizophrenia [25]. To summarize, cognitive dysfunction is a hallmark of schizophrenia that is increasingly being recognized [26] and should be taken into account when considering issues of etiology, course of illness, and treatment. Indeed, it has been argued that neuroimaging of cognitive dysfunction in schizophrenia will prove to be useful in characterizing the genetic and environmental risk factors that are likely to be causally related to the disorder [27].

In the treatment of cognitive dysfunction in schizophrenia, an important question is whether cognitive training can remediate impairments. Antipsychotic medication is not very successful in improving cognitive function, but neither does it deteriorate cognition, when adequately dosed [28-30]. Medication with weak dopamine antagonists could be associated with increased activation of frontal areas in schizophrenia patients [31]. Over the past years, several meta-analyses have shown that cognitive training can improve cognitive functioning in patients, above and beyond improvement on a specific cognitive task that was trained $[32,33]$. Cognitive training may consequently also improve brain function as measured with neuroimaging methods [34]. A typical cognitive training program consists of several weeks of training with several sessions a week in which the patient receives instruction to improve strategy and extensively practices computer-based cognitive tasks.

\section{Cognitive and Neural Basis of Hallucinations}

Hallucinations are a frequent symptom of psychosis and may occur (lifetime) in 60-70\% of patients with schizophrenia [35]. Although hallucinations can occur in any sensory modality (visual, auditory, somatosensory, olfactory, gustatory), auditory hallucinations are most common in schizophrenia. Specifically, most patients are bothered by auditory-verbal hallucinations (AVH), or "hearing voices." 
Typically, they hear disturbing comments on their behavior, or abusive language. Most hallucinations have a negative content and distract patients from their daily tasks. The frequency can differ widely, with some patients only hearing brief comments a few times a week to others with continuous hallucinations. Approximatly half of the patients experience the voices as coming from outside the head, the other half experiences them as coming from inside the head-but not being their own voice [36]. Antipsychotics are generally effective in reducing hallucinations but fail to reduce the symptom in up to $30 \%$ of patients. In order to measure brain activity that directly correlates with the ongoing experience of hallucinations, investigators using functional MRI have asked patients to indicate with the aid of button-presses the occurrence of hallucinations in the scanner. For this, patients are needed who experience intermittent hallucinatory episodes. This allows the investigator to contrast scans made during the hallucinatory episodes with those made in the absence of hallucinations. A drawback of this method is that only small groups can generally be included in such studies, as this method requires that patients experience several discrete periods of hallucinations during their stay in the scanner (typically an hour). Thus, the power of such studies tends to be limited, and their results should be interpreted with some caution. These studies typically involve the superior temporal gyrus in the left, but occasionally also right, hemisphere [3740]. Other cortical areas that have been shown to activate during hallucinations include the anterior cingulate cortex and the inferior frontal gyrus (e.g., Broca's area and its homologue in the right hemisphere). Activation of subcortical areas such as thalamus and basal ganglia has also been reported regularly. Two meta-analyses have summarized the brain activation studies on hallucination-related activation during scanning [41, 42]. The meta-analysis reported by Jardri et al. integrated findings from 10 studies, including a total of 68 patients diagnosed with a disorder in the schizophrenia spectrum. Analyses involved the stereotactic coordinates of 129 significant foci. AVH were associated with increased activation likelihoods in a bilateral neural network that included Broca's area, the anterior insula, the precentral gyrus, the frontal operculum, the middle and superior temporal gyri, the inferior parietal lobule, and the hippocam$\mathrm{pal} /$ parahippocampal region. Six out of eight activation clusters were found in the left hemisphere. The authors concluded that not only frontotemporal speech areas are involved in the mediation of $\mathrm{AVH}$, but also medial temporal areas associated with verbal memory. The other meta-analysis, by Kühn and Gallinat [42], distinguished between "state" versus "trait" aspects of AVH. "State" refers to the hallucinatory state, that is, the activation of brain regions during the experience of hallucinations (e.g., with the button-press method). "Trait," in contrast, refers to the comparison of brain activity between patients with and without hallucinations in the preceding week or month, and thus concerns the tendency to hallucinate or liability for hallucinations. Kühn and Gallinat [42] found that the state of experiencing AVH is primarily related to speech production regions such as Broca's area, whereas the trait that may render an individual prone to hallucinations is related to brain regions involved in auditory processing

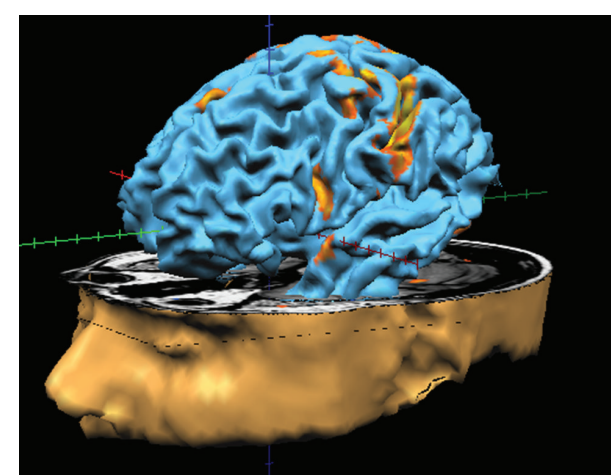

FIgURE 1: Activation of brain areas during language processing as measured with an fMRI scan (Philips 3T, Neuroimaging Center Groningen). Such language-related regions in the inferior frontal gyrus (Broca's area), temporoparietal cortex (Wernicke's area), and anterior cingulate are also seen to be active during auditory-verbal hallucinations.

and speech perception. Thus, aberrant activation of speech processing circuitry is essential to AVH (see Figure 1).

Treatment of hallucinations in schizophrenia consists mainly of prescription of antipsychotics. However, for the patients in which medication does not ameliorate hallucinations sufficiently (about $30 \%$ of patients with hallucinations), cognitive therapy can be useful. In addition, noninvasive neurostimulation by means of repetitive transcranial magnetic stimulation (rTMS) has been used to reduce hallucinations [43-45]. Most studies apply daily $1 \mathrm{~Hz}$ stimulation over the left temporoparietal junction for 15-30 min. during 1-3 weeks. Bilateral stimulation of the temporoparietal region has also been tried, but did not yield better effects [46]. A recent meta-analysis revealed a moderate effect size (in the order of $d=0.40$ ) for rTMS as compared to sham in improving auditory-verbal hallucinations in schizophrenia [47].

Research into the cognitive basis of hallucinations has revealed three factors to be of importance (for a recent overview of relevant cognitive processes, see [48]). One refers to the role of intrusions and the lack of cognitive inhibition. More specifically, studies that have used tasks demanding the volitional suppression of memory events and irrelevant memories have shown that AVH in schizophrenia are associated with a deficit in intentional cognitive inhibition [49-51]. A second factor concerns top-down influences on perception. Besides "bottom-up" influences (data coming in from the senses), perception is also guided by internally generated elements. These are referred to as "top-down factors." In bottom-up processes, the focus is on physical characteristics of a stimulus that drive perception. Top-down factors, in contrast, include prior knowledge, perceptual expectations, and mental imagery. The role of memory and thought in shaping perception is acknowledged. Put another way, this refers to processes that contribute to perception but do not originate directly from the external world but from the mind/brain of the perceiver. Several theoretical suggestions have been made implying an overreliance on top-down factors at the cost of bottom-up factors in driving hallucinatory perception (cf. [52]). For example, Grossberg [53] suggested 
that strong top-down excitation can create conscious experiences in the absence of bottom-up information. In this way, conscious mental imagery can arise. Grossberg then proposes a mechanism by which this top-down excitation becomes chronically hyperactive, through which sensory expectations can generate conscious experiences (through the activation of mental images) that are not under volitional control of the subject-in other words, hallucinations. The hypothesis that people with hallucinatory propensity may be characterized by strong top-down expectations has been put to the test empirically. Vercammen and Aleman [54] investigated the role of semantic expectations on perception in nonclinical individuals (i.e., without a psychiatric disorder or psychiatric symptoms that need treatment) with a disposition towards hallucination (as established with the revised Launay-Slade Hallucination Scale, LSHS). Studying people without a psychiatric disorder, but with a predisposition for hallucinations has several advantages. Specifically, the data are not contaminated by the possible effects of medication, hospitalization, or severity of psychopathology. The study involved 40 subjects selected from 350 undergraduates with the help of the LSHS to ensure sufficient variation regarding the experience of hallucination-like events (i.e., people with high scores and low scores were selected along with people with intermediate scores). Semantic expectation was manipulated by presenting sentences and leaving out the last word. That is, the last word was not clearly audible as it was embedded in white noise. Stimuli consisted of short sentences of 5-7 words, such as "The unfortunate carpenter hit his...." A predictable word would then be "thumb," but the actually presented word was "toe." Another example: "The thief reported to the....," where the word "owner" was presented instead of the more expected "police." The distinction between expected and unexpected words was validated in a separate pilot study. Subjects were asked to press the appropriate response button to indicate whether or not they heard a word and subsequently to identify this word out loud. Subjects were encouraged to identify the word only if they were positively convinced, and otherwise to state that they were uncertain of its identity. Results revealed that LSHS scores were correlated with the number of semantically primed errors. More specifically, subjects with higher levels of hallucination proneness were more likely to report hearing a word that fits the sentence context, when it was not actually presented. This effect remained significant after controlling of general performance on the task. Thus, these results support the conclusion that aberrant top-down processing, particularly in the form of strong semantic expectations, may contribute to the experience of auditory-verbal hallucinations.

In a follow-up study, Daalman et al. [55] investigated the same task for semantically primed top-down errors in patients with schizophrenia, people from the general population reporting auditory-verbal hallucinations, and healthy control subjects. They replicated the finding by Vercammen and Aleman [54] for the nonpsychotic individuals with AVH: they made significantly more top-down errors compared to healthy controls, while overall accuracy was similar. Notably, the severity of hallucination proneness correlated with the number of top-down errors. Thus, the results confirmed that nonpsychotic individuals with AVH are influenced more strongly by top-down processing (i.e., perceptual expectations) than healthy controls. In the patient group, however, the number of top-down errors, corrected for overall accuracy was in between those of the other two groups and did not differ significantly from either the nonpsychotic individuals with AVH or the healthy controls. The seemingly lack of a role for semantic expectations in the patient group suggests different cognitive mechanisms for pathological and nonpathological hallucinations, which not only calls for further comparison of cognitive mechanisms underlying different forms of hallucination, but also challenges the notion of a continuity between psychotic-like experiences in people without a psychiatric diagnosis and psychotic phenomena in patients [56].

With regard to the neural basis of top-down processing, secondary perceptual areas are of importance. A study of verbal speech imagery [57] revealed activation of speech perception cortex (Wernicke's area). Activation in such areas has also been shown to be strong during hallucinations (as reviewed above). Interestingly, the temporoparietal region in the left hemisphere (converging with Wernicke's area) has been shown to have reduced connectivity in the resting states to the cingulate cortex and the bilateral amygdala [58]. The cingulate has been shown to play a key role in self-referent processing and attentional control (monitoring), whereas the temporoparietal cortex has been implied in sense of agency and the amygdalae in emotional salience. Finally, a study using functional magnetic resonance imaging with dynamic causal modeling for the analysis of directionality in activated networks revealed reduced connectivity from Wernicke's to Broca's area in patients with auditory-verbal hallucinations [59]. This may suggest that activity in Broca's area may be less constrained by perceptual information received from the temporal cortex and Broca may subsequently actively generate spurious activity in a search for meaningful linguistic percepts.

The third, and most researched, cognitive factor involved in hallucinations concerns source-monitoring. This refers to the ability to discriminate between imagined and perceived information, that is, between internal and external sources driving the perceptual experience [60]. More specifically, it has been suggested that a difficulty to differentiate between information that has been perceived from the outside world and information that has been imagined may explain the genesis of hallucinations [61]. These symptoms suggest that the patient has difficulty in maintaining the distinction between internally- and externally-generated events, which have been referred to as manifestations of autonoetic agnosia, meaning literally "the inability to identify self-generated mental events" [62]. Therefore, these symptoms could be caused by a deficit in monitoring the self-generation of thoughts [63] or a bias towards misattributing internal thoughts to external sources [64].

Autonoetic agnosia has been measured with various cognitive tests, including the assessment of source monitoring [65]. A meta-analysis of studies investigating accuracy of self-attribution in schizophrenia revealed a significant impairment in patients as compared to age and education matched control subjects [66]. There was also a relationship 
with hallucinations: patients with hallucinations made more errors than patients without hallucinations. The systematic review included 23 published studies that contrasted the performance of schizophrenia patients with healthy controls (with a total of 1370 participants) and 9 studies that directly compared patients with and without auditory hallucinations (315 participants). The authors noted that the homogeneity in results across studies was remarkable. That is, the selfrecognition difficulties were found regardless of the action modality, timing delay, and design used to measure selfrecognition. Some tasks involved voice recognition, others source memory for words or pictures.

The neural basis of source monitoring has also been studied. For example, Simons et al. [67] used fMRI to investigate the brain areas associated with this ability in healthy subjects, who were to remember whether information had previously been perceived or imagined, or whether information had been presented on the left or right of a monitor screen (control condition). Their results indicated a role of the medial anterior prefrontal cortex, thalamus, and cerebellum in reality monitoring. These regions have also been implicated in schizophrenia. Morever, reduced prefrontal activation was associated with the same misattribution error that has been observed in schizophrenia. Allen et al. [68] investigated neural activation differences between patients with and without hallucinations during a verbal self-monitoring task. They hypothesized that patients with a history of auditory verbal hallucinations would misattribute their own speech as external and show differential activation in brain areas implicated in hallucinations. Subjects listened to prerecorded words while being scanned with fMRI. The source (self/nonself) and acoustic quality (undistorted/distorted) of the presented words were varied across trials. The hallucinator group made more external misattributions (indicating that their own, slightly distorted speech was that of another person) and showed altered activation in the superior temporal gyrus and anterior cingulate compared with both other groups. The authors interpreted the reduced activation of the anterior cingulate as evidence it impaired self-monitoring.

\section{Emotional Processing Abnormalities: Perception and Regulation}

Although several authors have maintained that schizophrenia is primarily a cognitive disorder $[26,69,70]$, research in recent decades has been accumulating to certainly also support the view of schizophrenia as an emotional disorder [71, 72]. Indeed, this may seem surprising as schizophrenia has always been considered to be a "nonaffective" psychosis in psychiatry. However, the term "nonaffective" was primarily used to indicate that mood disturbance is not a key feature of schizophrenia, in contrast to bipolar disorder or psychotic depression. The evidence for emotional abnormalities in schizophrenia comes from studies investigating emotional perception, emotional experience, and emotion regulation. A large and robust deficit in emotion perception was quantified for patients with schizophrenia, irrespective of task type, in a meta-analysis of facial emotion perception studies [73]. Not only emotion perception from facial expressions is impaired, recognition of emotional prosody (from tone of voice) is equally impaired [74]. A meta-analysis of studies investigating deficits in emotion perception and their relationship to outcome measures documented a significant association between poor emotion perception and worse outcome in schizophrenia [75]. The authors included 25 studies and reported medium to large range positive correlations between emotion identification and functional outcome domains involving social problem solving, social skills, and community functioning. Li et al. [76] reported a meta-analysis of 15 activation studies during facial emotion processing in schizophrenia and found reduced activation in bilateral amygdala, parahippocampal gyrus, and fusiform gyrus, right superior frontal gyrus and lentiform nucleus of patients as compared to healthy controls. In contrast, Anticevic et al. [77] reported a meta-analysis of neuroimaging studies that investigated amygdala recruitment in response to aversive emotional stimuli in schizophrenia and reported that patients activate the amygdala similarly to healthy controls. This could suggest that the emotion perception deficit may be more related to early processing deficits in perceptual regions or late processing deficits in association with cortex or frontal regions (involved in categorization) than to diminished responsivity of emotional circuitry, though this remains a question for further research. Notably, in a study of social perception, in which subjects rated the trustworthiness of faces during fMRI, we observed reduced amygdala activation to untrusworthy faces in patients with schizophrenia as compared to age- and education-matched healthy control subjects [78]. Thus, in certain circumstances the amygdala response might be compromised in schizophrenia, maybe especially when subtle social cues are involved. The studies using aversive emotional stimulation generally used very explicit and strong negative stimuli.

Recent emerging work with functional imaging is providing key insights in the neural correlates of cognitive influences on the emotion control. Ochsner et al. [79] examined the ability to cognitively transform the emotional experience of a negative affect, which has been termed "reappraisal." Generating a more positive interpretation (or less negative interpretation) for an image showing people in pain, which initially triggers negative affect, is an instance of reappraisal. Strategies people can use to reappraise involve telling themselves that people in the photo are not really in that much trouble, that things will not turn out all that badly, or that things are not quite as bad as they seem [80]. In case human injury is portrayed one could imagine that it is a scene from a movie and no real injury was involved, and so forth. Numerous psychological studies have shown this to be an effective way of reducing emotion-induced distress [81]. Ochsner et al. [79] proposed that reappraisal would be mediated by interactions between cognitive control and emotionprocessing systems. Concerning cognitive-processing systems, they proposed the involvement of three major areas: (1) the LPFC, implicated in generating a strategy for cognitively reframing an emotional event in unemotional terms and keeping that in mind by working memory processes; (2) the dorsal anterior cingulate cortex, regarding the interference between top-down reappraisals that neutralize affect and 
bottom-up evaluations that continue to generate an affective response and consequently signaling the need for reappraisal to continue; and (3) the dorsal regions of the MPFC, involved in reevaluating the relationship between internal states and external stimuli used to monitor changes in one's emotional state during reappraisal. With respect to emotion-processing systems, the amygdala and medial orbital frontal cortex (MOFC) are associated with differentially encoding and representing the affective properties of stimuli [82].

Participants in Ochsner et al's [79] study were shown aversive and neutral photos in order to compare reappraisal to another condition that could draw on processes invoked by reappraisal but which would not be related to regulation of affect per se. For that purpose, they employed two conditions: (1) "attend", where participants were asked to let themselves respond emotionally to each photo by being aware of their feelings without trying to alter them and (2) "reappraise", where subjects had to interpret photos in such a way that they felt less negative in response to them. As both conditions involved attention to emotion, regions with greater activation when reappraising than attending were thought to reflect processes used to exert cognitive control. In contrast, regions more active for attend than reappraise trials were hypothesized to be important for emotion processing that would be deactivated by reappraisal. According to their findings, reappraisal of negative photos successfully diminished negative affect, and the neural correlates were (1) activation in the regions of the LPFC and MPFC essential for working memory, cognitive control, and self-monitoring and (2) decreased activation in the MOFC and the amygdala, which are involved in emotion processing [79]. This has subsequently been confirmed in other studies, although the decrease in amygdala activation was only reported in half of the studies. Nevertheless, a recent meta-analysis of 48 neuroimaging studies reported that reappraisal consistently activated cognitive control regions in frontal cortex and lateral temporal cortex and modulated the bilateral amygdala [83].

We investigated the neural correlates of reappraisal in people with a predisposition towards psychosis. To this end, 600 undergraduate students completed the Community Assessment of Psychic Experiences Questionnaire (CAPE), positive symptom subscale. We subsequently selected two groups, one from each extreme end of the distribution (total $N=34$ ). We expected less effective emotion regulation strategies in the high psychosis proneness group, but observed equal performance, that is, both groups reported successful diminishment of experienced negative emotion. However, high psychosis-prone subjects showed stronger activation than low subjects in a number of prefrontal regions (anterior cingulate, left dorsomedial PFC, and right ventrolateral PFC) during reappraisal, relative to only attending to negative pictures. The amygdala response to negative stimuli was decreased through reappraisal only in the low group. Functional connectivity analysis revealed less prefrontal-amygdala coupling in high psychosis-prone subjects. Thus, the evidence from neural activation patterns points towards less efficient interaction between regions involved in cognitive control and emotion processing. In patients with schizophrenia, reduced levels of reappraisal have been reported $[84,85]$, suggesting dysfunction in regions subserving cognitive control of emotion. However, only one study has been reported as yet using the above mentioned neuroimaging task in patients [86]. Schizophrenia patients showed that prefrontal hypoactivation of the right VLPFC occurred during downregulation, compared to control subjects, and showed no coupling between prefrontal cortex and amygdala, in contrast to control subjects.

\section{Social Cognition, Theory of Mind, and Empathy}

Social cognition concerns the cognitive functions underlying social perception and interaction. Besides emotion recognition, understanding other people is crucial for this. Theory of mind (ToM) can be defined as the ability to correctly attribute feelings, knowledge, intentions, and goals to other people and is of crucial importance for proper social interaction [87]. It has been suggested to be involved in positive symptoms of schizophrenia, for example, paranoid delusions [88, 89]. A deficit in overruling the automatic response and hence wrongfully maintaining a positive self-image may result in an impaired ToM, leading to an inappropriate attribution of threatening thoughts, intentions, or behaviors to others. Two main brain regions are found to play a crucial part in ToM, the temporoparietal junction and the prefrontal cortex. The temporoparietal junction is thought to be recruited selectively for the attribution of mental states to others [90, 91]. Furthermore, several neuroimaging studies have shown frontal lobe activation in healthy subjects while reasoning about other people's mental states (e.g., [92]) as well as an impaired theory of mind in people with frontal lobe lesions (e.g., [93]). At least three possible contributions of the frontal lobe to the ability to have a theory of mind have been formulated. First, the frontal lobe is thought to contribute to the theory of mind concerning the control processes supporting complex reasoning [94]. Second, the frontal lobes may be involved in holding two separate perspectives simultaneously [95]. A third possible contribution of the frontal lobes to theory of mind was put forward by Ruby and Decety [92]. They suggest that the frontal lobes are of crucial importance in inhibiting one's own perspective of the world while inferring someone else's perspective.

In an experiment by Vogeley and colleagues [96], short stories were administered in which subjects themselves were featured (high self-perspective), compared with stories in which the subject did not play any part (low self-perspective). In both types of stories, the subject had to infer the perspective of another person. However, only in the high self-perspective condition, the subject had to take his/her own perspective into account. This enabled the isolation of inhibition of one's own perspective and showed right inferior frontal gyrus activation. Consistent with these findings, Samson et al. [91, 94] developed an elegant paradigm which enables the direct comparison of a low inhibition selfperspective condition with a high inhibition self-perspective condition. This task is particularly interesting since it allows for the selective measurement of the inhibition of self-perspective in a nonemotional setting. The task was 
administered to a patient who suffered from a right hemisphere stroke, resulting in a lesion in the right inferior and middle frontal gyrus extending to the right superior temporal gyrus and showed a specific deficit in the high inhibition self-perspective task as compared to the low-inhibition selfperspective task. Following these results, Samson et al. [94] suggest that theory of mind consists of two components, which are confounded in classic theory of mind tasks. They propose that taking someone else's perspective may be a serial process in which the inhibition of self-perspective is a first and necessary step before someone else's perspective can be inferred. The brain region that is thought to be of critical importance for this process is the right inferior prefrontal cortex. This inference is confirmed by the findings that the right inferior prefrontal cortex is necessary for the inhibition of responses in nonsocial tasks [97].

With regard to insight, it could be hypothesized that due to a lack of inhibitory control rational, explicit self-processing cannot proceed and will therefore not be able to overrule the automatic response preserving the positive self-image. Psychotic patients who lack insight may show an impaired self-inhibition as compared to psychotic patients who do have insight into their condition, leading to the inability to use the feedback provided by the environment to adapt their wrongfully preserved self-image.

Theory of mind may further be decomposed into different mechanisms for the cognitive and the affective aspects of mentalizing, which may be differentially impaired in schizophrenia patients [98]. In order to study the role of PFC cortices for these different ToM aspects, Shamay-Tsoory and Aharon-Peretz [99] designed an interesting task that allowed evaluation of affective and cognitive mentalizing abilities based on verbal and nonverbal information. The task involves the ability to judge mental states based on verbal and eye gaze cues, with stimuli divided into three main conditions: affective, cognitive, and physical. Additionally, different aspects of ToM can be assessed: (1) first order ToM, requiring the attribution of a mental state (cognitive) or an emotion (affective) to a character and (2) second order ToM, based on understanding beliefs about others' beliefs and desires (cognitive) or understanding emotions about others' emotions (affective). Results showed that patients with schizophrenia (especially those with negative symptoms) and patients with ventromedial (VM) prefrontal damage were significantly less accurate in their response to affective 2 nd order ToM conditions compared to controls. We investigated differences in prefrontal cortex activation between subjects with high versus low psychosis proneness scores during mentalizing to further delineate the role of PFC regions in vulnerability towards psychosis. Using the same task as Shamay-Tsoory and Aharon-Peretz [99], we observed stronger prefrontal activation in psychosis-prone subjects than in subjects without this proneness during first and second order mentalizing [100]. For first order mentalizing, this excessive recruitment of PFC was localized in the anterior prefrontal cortex (BA 10 ), whereas for second order mentalizing lateral prefrontal regions were hyperactivated (BA 46/49). A limitation of the task may be that the stimuli are rather artificial, schematic drawings, which may compromise ecological validity.
Other ToM paradigms allow for measurement of inhibition of self-perspective, a key component of ToM, and belief reasoning being another important component. Samson et al. [94] showed that inhibition of self-perspective can be selectively impaired in patients with frontal brain damage. Using neuroimaging in healthy volunteers, with the same theory of mind task as Samson et al. [94], we found activation of bilateral inferior frontal gyrus for inhibition of self-perspective, while belief reasoning was mediated by the left superior and middle temporal gyri [101]. We also investigated brain activation during this task in participants with psychosis-proneness as established by high scores on the CAPE psychosis subscale [102]. A stop-signal task was included additionally, to measure simple response inhibition. Thus, we could test the hypothesis that the reduced inhibition of self-perspective is not due to a general reduction in the inhibitory function of the frontal cortex. A stronger activation of the left inferior frontal gyrus was found for psychosis-prone subjects, when compared to subjects without psychosis-proneness. Such a difference was not observed on the simple response inhibition task, however. Therefore, the results indicate that, at a neural level, psychosis-prone subjects need a stronger recruitment of neural resources in order to inhibit self-perspective. Indeed, the distinction between self-inhibition and belief reasoning needs further investigation in patient populations.

Research has also been directed at a concept that is related to ToM: empathy. Rather than concerning the ability to understand what others think, empathy refers to the ability to feel what others feel. Several studies have reported reduced levels of empathy in patients with schizophrenia [103]. Social cognition also encompasses other processes than the higherorder cognitive processes involved in theory of mind. More specifically, the investigation of perception and interpretation of social cues deserves more attention. For example, patients with schizophrenia may be impaired in detecting facial cues associated with trustworthiness [78, 104]. Other aspects of social cues involve signals of approach and avoidance. In schizophrenia, perception of such cues has been tested using a visual distance illusion in which two cartoon figures that are running and looking towards each other look more close to each other than two figures running and looking away from each other, even when the actual physical distance is the same $[105,106]$. Patients with schizophrenia are less sensitive to this illusion [106], as are patients with autism [105] and Klinefelter syndrome [106], who also have social-emotional deficits.

\section{Reduced Insight/Awareness of Illness}

Lack of insight (unawareness of illness) is a common and clinically relevant feature of psychosis [107]. It can be subdivided into three components: (1) awareness of having an illness, (2) recognizing psychotic symptoms as abnormal, and (3) acceptance of prescribed treatment [108]. Lack of insight has been associated with poor treatment compliance and medical adherence, poorer global functioning, severity of psychopathology, recurrence, and poorer outcome [109]. This is not only inconvenient for the patient, it can also lead to considerable distress in family members, friends and treating clinicians since it hampers their attempt to help and impedes 
with solutions to the problems engendered by the symptoms of the patient (e.g., believes he is being poisoned and refuses to eat). The cognitive and neural bases of insight in psychosis remain unclear, however, rendering it a scientific mystery. Although associations have been established between poor insight in psychosis and reduced cognitive functioning, especially with regard to cognitive set-shifting thought to be mediated by the frontal cortex, the modest magnitude of the association suggests that cognitive factors can not sufficiently explain impaired insight (see meta-analysis by Aleman et al., [110]). Indeed, in a replication and extension of this metaanalysis (based on data from 72 studies and a total population of 5429 patients), Nair et al. [111] reported that there is a small but significant relationship between clinical insight, some aspects of cognitive insight, and neurocognition. This underlines the complexity of the insight construct and may imply that while the neurocognitive model is important, cognitive impairment is likely to be the only one of several contributing factors. A study by Quee et al. [112] in 270 patients with nonaffective psychosis points to one of these factors: phase of illness. Neurocognitive impairment was associated with reduced insight, but only in patients with multiple episode or chronic psychosis. Social cognition, that is, recognition of emotional expressions and mentalizing, was also related to insight. No relationship between cognitive impairment and reduced insight was found in patients with recent-onset psychosis.

A framework is needed that can account for the above mentioned observations, that is, the role of impaired cognitive flexibility and other cognitive impairments, but that at the same time moves beyond these factors, as they can only provide partial explanations at best. Insight in psychosis could be hypothesized to depend on self-evaluation, where self-evaluation is conceptualized along the lines of Epstein's [113] cognitive-experiential self theory (CEST). According to CEST, people respond to the world on two levels, rational and experiential. These ways of responding are conceived of as two distinct mental systems for adapting to the demands of our environment. The rational system is thought to operate primarily on a conscious level, involving working memory and language (though the latter not by definition). In contrast, the experiential self has a stronger emotional input. It is thought to operate largely at an unconscious level and processes information fast and holistically. The rational system will be slower than the experiential system and will require more effort. It is important to note that, although the two systems are thought of as independent, they are assumed to interact with each other and will both influence behavior. This account can be complemented by the model of dual attitudes, proposed by Wilson et al. [114]. This model highlights the complex interplay between implicit and explicit evaluations and posits that people may simultaneously possess different evaluations towards the same attitude object. Such dual attitudes will then become represented at an explicit level, where they are readily accessible to self-reporting. However, the activation of these newly formed attitudes has not yet become automatic and, hence, requires a fair amount of cognitive capacity and motivation. At the same time, people's original attitudes are assumed to continue to exist in memory, albeit at an implicit level. When there is sufficient capacity and motivation, people will effortfully retrieve their newly formed, explicit attitudes from memory. By contrast, when capacity and motivation are lacking, people's original, implicit attitudes may reemerge. It is important to note that several quantitative measures have been developed to target aspects of implicit and explicit self-processing (e.g., $[114,115])$.

In short, consistent with CEST, one can presume that implicit, experiential self-processing is biased towards maintaining a positive self-image (which excludes severe mental illness). To obtain insight in psychosis, rational, explicit selfprocessing must overrule this automatic response. This will only occur in the face of sufficient cognitive capacity and motivation. However, a large number of psychotic patients lack both, which hampers the development of insight.

A number of studies have focused on the hypothesis that reduced insight may arise from abnormalities in brain circuits subserving self-evaluation. Self-evaluation involves the ability to think about and make judgments regarding one's own cognitive, personality, physical, and emotional characteristics. Thus, patients with reduced insight are hypothesized to have difficulties in evaluating their own behavior and mental processes in relationship to their environment, and to implicit and explicit sociocultural rules and expectations. An important component of self-awareness is the ability to reflect on your personal abilities, characteristics, and personal situation. The underlying neurocognitive processes for such self-evaluation are still unclear but potentially relevant for understanding insight in psychosis. Johnson et al. [116] studied brain activation during self-evaluation in healthy subjects, who were asked to respond to a variety of statements requiring knowledge of and reflection on their own abilities, traits, and attitudes (e.g., "I forget important things", "I am a good friend"). Reponses were compared to those of statements requiring a basic level of semantic knowledge (e.g., "ten seconds is more than a minute", "you need water to live"), used as a control condition for auditory comprehension, attentional demands, decision-making, the motoric response, and any common retrieval processes. The results showed involvement of the medial prefrontal cortex and posterior cingulate during self-evaluation. This is consistent with lesion locations in patients with impaired self-awareness, as well as with other functional imaging studies involving mentalizing about the self or others [117]. Thus, one could hypothesize reduced activation of self-related regions in patients with poor insight as compared to patients with intact insight.

Van der Meer et al. [118] provided a quantitative integration of the neuroimaging evidence regarding self-evaluation, which yielded two areas within the medial prefrontal cortex (MPFC), namely the ventral and dorsal MPFC. In a study that contrasted schizophrenia patients with good versus poor insight on a task of self-evaluation and other evaluations, better insight (as measured with the Schedule of Assessment of Insight Expanded [119]) was associated with greater response in the inferior frontal gyrus, anterior insula, and inferior parietal lobule during self-reflection [120]. In addition, better cognitive insight (as measured with the Beck Cognitive Insight Scale [121]) was associated with higher activation in the ventromedial prefrontal cortex during self-reflection. 
The cognitive regulation of emotion may also be regarded to be of relevance for insight. Patients with reduced insight seem to have more difficulties in accepting the emotional consequences of a threatening experience to the self, as is becoming aware of having a mental illness. Several studies have found that many forms of psychopathology revolve around failures to adaptively regulate emotional responses, with consequences ranging from personal distress to socially maladaptive and self-destructive behaviors [122]. Empathy may also be of relevance. We hypothesized a relationship between reduced levels of empathy and less insight in psychosis, based on the rationale that being touched by the emotional state of others will facilitate the accommodation of their perspective and will therefore make a contribution to insight over and above emotion perception and perspective taking. Consistent with this prediction, a regression analysis involving several cognitive and affective measures showed the strongest unique contribution to be from empathy [123]. For this study, fortysix patients with a diagnosis of schizophrenia and fifty-three healthy controls were assessed with a test battery consisting of tests of social cognition (a self-rating scale for affective empathy, a ToM task assessing both cognitive and affective ToM, and two tests of emotion perception), verbal memory, executive functioning, psychomotor speed, and intelligence. Being able to empathize with others at both the affective and cognitive level may enhance insight in schizophrenia. Brain research could focus on regions known to be involved in empathy, for example, anterior insula, cingulate cortex, and regions subserving action simulation and mentalizing [124].

\section{Apathy}

Besides psychotic symptoms such as hallucinations on the one hand and cognitive impairment on the other, lack of initiative and social withdrawal are key symptoms of schizophrenia. Apathy or lack of will (also termed avolition or abulia) refers to markedly reduced levels of interest in daily activities and a lack of initiative. It may present as indifference. Profound levels of apathy are common in schizophrenia patients, and have been suggested to be the most central feature of negative symptoms, representing the critical component, particularly with regard to functional outcome [125]. Understanding apathy has important implications, as apathy is the strongest predictor of poor functioning, unemployment, severity of illness, and worse outcome in routine clinical practice $[126,127]$. Indeed, apathy is arguably one of the most important clinical and scientific problem for schizophrenia researchers to tackle. Surprisingly, though, studies into the cognitive and neural substrate of apathy are scarce, even though a number of studies have addressed the brain basis of negative symptoms of schizophrenia, that largely reflect apathy (e.g., affective flattening, diminished levels of interest, social withdrawal, and lack of energy). However, there is also a paucity of research into the cognitive and neural basis of negative symptoms in general. Positive symptoms, such as delusions and hallucinations, may dominate in acute phases of the illness and draw more attention due to their bizarre nature. In addition, there has been a lack of theorizing with regard to negative symptoms such as apathy. Apathy should be studied in its own right. Indeed, accumulating evidence supports apathy as a distinct syndrome with an identifiable neurobiology [128].

Different forms of apathy have been distinguished on clinical grounds. For example, Stuss et al. [129] proposed multiple subtypes, depending on which neural circuit is affected. Involvement of the oculomotor circuit would yield a different form of apathy (e.g., based on neglect) than the involvement of the supplementary motor circuit (reduced motor output). They also distinguished between apathy as a consequence of orbitofrontal impairment, which would be associated with a reduction in motivational response due to lack of limbicaffective input and "social apathy," which would result from disturbance in sense of self and social awareness due to anterior frontal lesions. Levy and Dubois [130] distinguished between three subtypes: "emotional-affective," "cognitive," and "auto-activation." The first would be characterized by deficient linkage of emotional-affective signals and ongoing behavior, the second by difficulties in elaborating action plans necessary for goal-directed behavior, and the third by the inability to self-activate thoughts and actions. The different forms of apathy are not primarily due to depression, but like depression, involve a reduction in behavioral activation.

The conceptualisation of different types of apathy has not been taken to schizophrenia as yet, but research is ongoing at the University Medical Center Groningen to investigate this in detail. Initial evidence for apathy subtypes in schizophrenia receives support from factor analyses on the structure of negative symptoms. Negative symptoms of schizophrenia are normally grouped into a single category. We recently carried out two factor analyses on large independent data sets that confirmed the distinction between two factors [131]. First, an exploratory factor analysis was carried out based on interviews with the Positive and Negative Syndrome Scale (PANSS) in a sample of 664 schizophrenia patients from the north of the Netherlands (provinces Groningen, Friesland, and Drenthe). Only items related to negative symptoms in earlier studies were included and categorized into one or more negative symptom dimensions. In a second step, the acquired symptom structure was subsequently tested for stability by confirmatory factor analysis of PANSS interviews in a large separate cohort of 2172 schizophrenia patients, from the GROUP study, that involves 13 research centers from all regions in the Netherlands. Item selection and exploratory factor analysis resulted in a two-factor structure of negative symptoms. The first factor consisted of PANSS items N1 flat affect (lack of expressivity), N3 poor rapport, N6 lack of spontaneity, G5 mannerisms and posturing, G7 motor retardation and G13 avolition. The second factor consisted of items N2 emotional withdrawal, N4 passive/apathetic social withdrawal, and G16 active social avoidance.

We interpreted the first factor as being related to core negative symptoms of schizophrenia, reflecting loss of initiation of action. It could be described as a "loss of initiative" factor. The second factor could be described as a socialemotional withdrawal factor, in particular "loss of interest" in these areas. Apathy is central to most of these items (e.g., avolition or passive/apathetic social withdrawal), which supports extrapolation to apathy proper. 
Depression can pose a source of clinical and conceptual confusion, as it may be difficult to differentiate it from apathy. However, although apathy can be an expression of depression, it is independent from the symptoms associated with depression [130]. For example, key symptoms of depression as defined by the World Health Organization's international classification of diseases (ICD) include loss of confidence, excessive guilt, recurrent thoughts of death, poor concentration, sleep disturbance, and change in appetite or weight. These symptoms are not part of the concept of apathy, although some of them may be correlated, such as poor concentration. Indeed, the apathy evaluation scale [131] has been shown to correlate only weakly with depression. To avoid confusion through possible overlap with clinical depression, it is advisable to exclude a diagnosis of depression (defined as DSM-IV major depressive disorder, dysthymic disorder, depressive disorder not otherwise specified or bipolar disorder) in patients that are included in studies of apathy in schizophrenia, Parkinson's Disease or Alzheimer's Disease.

One could hypothesize different neural circuits to underlie different forms of apathy. For example, a lack of initiative could be linked to a deficient frontoparietal action network, whereas a lack of interest could be linked to a deficient ventral striatal-orbital-medial frontal saliency network. Few studies have as yet investigated the neural basis of apathy in schizophrenia, however. One study reported reduced frontal lobe volumes and more pronounced cognitive deficits in schizophrenia patients with high levels of apathy compared to schizophrenia patients with low levels of apathy [132]. Callicott et al. [133] reported an association between negative symptoms and reduced levels of $\mathrm{N}$-acetyl-aspartate in DLPFC, using magnetic resonance spectroscopy, suggesting reduced neuronal integrity in that area. With regard to motivational processing, a recent fMRI study [134] showed that higher anhedonia was associated with reduced activation to positive versus negative stimuli in bilateral amygdala and right ventral striatum in patients with schizophrenia. In a similar vein, Simon et al. [135] recently reported a negative correlation between apathy levels and ventral-striatal activation during reward anticipation in schizophrenia patients, while activation during receipt of reward was negatively correlated with severity of depressive symptoms. These studies did not distinguish between subtypes of apathy, however, which are in need of further investigation.

In terms of treatment, apathy is notoriously difficult to improve. Novel pharmacotherapeutical approaches are being tested, such as sarcosine, a compound that targets glutamatergic transmission in the frontal cortex. Sarcosine is a selective inhibitor of the glycine transporter 1 [GlyT1] that has shown promise in improving negative symptoms of schizophrenia [136]. Other compounds that modulate glutamatergic signalling are being investigated [137]. Noninvasive neurostimulation with rTMS may also be a promising treatment option. Most studies apply daily $10 \mathrm{~Hz}$ stimulation over the left dorsolateral prefrontal cortex. A meta-analysis of nine trials published by 2008 yielded a significant effect (with moderate effect size) of rTMS as compared to sham TMS in improving negative symptoms [138]. Studies with daily stimulation for three weeks or more showed a larger effect size than trials of shorter duration. This was confirmed recently in a metaanalysis that included 12 studies, published up to 2011 [139]. It would be of interest to use fMRI before and after rTMS trials for improving apathy to evaluate whether increased activation of the dorsolateral prefrontal cortex can account for clinical improvement. Future research will also benefit from the development of brief, reliable, and valid standardized measures of negative symptoms (especially apathy/avolition) which will aid its assessment in multicenter trials [140].

\section{Concluding Remarks}

Although etiology and treatment of schizophrenia remain poorly understood, recent decades have shown progress in delineating cognitive and emotional processing abnormalities and elucidating their neural basis. Impaired activation of and connectivity between frontotemporal, frontoparietal, and frontostriatal brain networks subserving cognitive functioning and integration of cognition and emotion has been consistently reported. A distributed network of cortical and subcortical regions is activated during hallucinations [141]. Language circuitry has been shown to be involved in auditory-verbal hallucinations, and rTMS over language regions may reduce severity of hallucinations in some patients. The effects on brain activation of such treatments deserve further investigation [142]. Top-down attentional processing in perception may be dysregulated in people with a disposition towards hallucination. Perception of emotion is impaired in schizophrenia patients and emotion regulation may be compromised due to reduced cognitive control of emotion (connectivity between frontal areas and limbic areas). Social cognition and especially theory of mind processes have been the subject of intensive study. These processes are highly relevant for social functioning, an important target outcome for treatment of schizophrenia. Impaired selfevaluation, mediated by midline cortical areas (medial frontal and posterior cingulate) may contribute to poor awareness of illness, and more specifically insight into psychosis. Finally, future research should focus on pervasive negative symptoms such as apathy and underlying neural systems. This will pave the way for better treatment options and ultimately a better quality of life for people suffering from schizophrenia.

\section{Conflict of Interests}

The author declares that there is no conflict of interests regarding the publication of this paper.

\section{Acknowledgment}

The author was supported by a VICI Grant no. 016.128.606 from the Netherlands Organisation for Scientific Research (N.W.O.).

\section{References}

[1] K. T. Mueser and S. R. McGurk, "Schizophrenia," The Lancet, vol. 363, no. 9426, pp. 2063-2072, 2004. 
[2] A. Jablensky, "Schizophrenia: the epidemiological horizon," in Schizophrenia, S. R. Hirsch and D. R. Weinberger, Eds., Blackwell, Oxford, UK, 1995.

[3] H. I. Kaplan, B. J. Sadock, and J. A. Grebb, Synopsis of Psychiatry, William and Wilkins, Baltimore, Md, USA, 1994.

[4] A. Aleman, R. S. Kahn, and J.-P. Selten, "Sex differences in the risk of schizophrenia: evidence from meta-analysis," Archives of General Psychiatry, vol. 60, no. 6, pp. 565-571, 2003.

[5] The American Psychiatric Association, Diagnostic and Statistical Manual of Mental Disorders, The American Psychiatric Association, Arlington, Va, USA, 5th edition, 2013.

[6] W. T. Carpenter Jr., J. S. Strauss, and S. Muleh, "Are there pathognomonic symptoms in schizophrenia? An empiric investigation of Schneider's first rank symptoms," Archives of General Psychiatry, vol. 28, no. 6, pp. 847-852, 1973.

[7] N. C. Andreasen, M. Flaum, V. W. Swayze II, G. Tyrrell, and S. Arndt, "Positive and negative symptoms in schizophrenia: a critical reappraisal," Archives of General Psychiatry, vol. 47, no. 7, pp. 615-621, 1990.

[8] H. Häfner and W. An der Heiden, "Course and outcome of schizophrenia," in Schizophrenia, S. R. Hirsch and D. R. Weinberger, Eds., Blackwell, London, UK, 2nd edition, 2003.

[9] R. W. Heinrichs and K. K. Zakzanis, "Neurocognitive deficit in schizophrenia: a quantitative review of the evidence," Neuropsychology, vol. 12, no. 3, pp. 426-445, 1998.

[10] A. Reichenberg and P. D. Harvey, "Neuropsychological impairments in schizophrenia: integration of performance-based and brain imaging findings," Psychological Bulletin, vol. 133, no. 5, pp. 833-858, 2007.

[11] A. Aleman, R. Hijman, E. H. F. De Haan, and R. S. Kahn, "Memory impairment in schizophrenia: a meta-analysis," The American Journal of Psychiatry, vol. 156, no. 9, pp. 1358-1366, 1999.

[12] J. Schaefer, E. Giangrande, D. R. Weinberger, and D. Dickinson, "The global cognitive impairment in schizophrenia: consistent over decades and around the world," Schizophrenia Research, vol. 150, no. 1, pp. 42-50, 2013.

[13] M. M. Sitskoorn, A. Aleman, S. J. H. Ebisch, M. C. M. Appels, and R. S. Kahn, "Cognitive deficits in relatives of patients with schizophrenia: a meta-analysis," Schizophrenia Research, vol. 71, no. 2-3, pp. 285-295, 2004.

[14] B. E. Snitz, A. W. MacDonald III, and C. S. Carter, "Cognitive deficits in unaffected first-degree relatives of schizophrenia patients: a meta-analytic review of putative endophenotypes," Schizophrenia Bulletin, vol. 32, no. 1, pp. 179-194, 2006.

[15] D. Dickinson, M. E. Ramsey, and J. M. Gold, “Overlooking the obvious: a meta-analytic comparison of digit symbol coding tasks and other cognitive measures in schizophrenia," Archives of General Psychiatry, vol. 64, no. 5, pp. 532-542, 2007.

[16] E. E. M. Knowles, A. S. David, and A. Reichenberg, "Processing speed deficits in schizophrenia: reexamining the evidence," The American Journal of Psychiatry, vol. 167, no. 7, pp. 828-835, 2010.

[17] S. R. Marder and W. Fenton, "Measurement and treatment research to improve cognition in schizophrenia: NIMH MATRICS initiative to support the development of agents for improving cognition in schizophrenia," Schizophrenia Research, vol. 72, no. 1, pp. 5-9, 2004.

[18] B. W. Palmer, R. K. Heaton, J. Kuck et al., "Is it possible to be schizophrenic yet neuropsychologically normal?” Neuropsychology, vol. 11, no. 3, pp. 437-446, 1997.
[19] C. M. Wilk, J. M. Gold, R. P. McMahon, K. Humber, V. N. Iannone, and R. W. Buchanan, "No, it is not possible to be schizophrenic yet neuropsychologically normal," Neuropsychology, vol. 19, no. 6, pp. 778-786, 2005.

[20] D. R. Weinberger, K. F. Berman, and R. F. Zec, "Physiologic dysfunction of dorsolateral prefrontal cortex in schizophrenia. I. Regional cerebral blood flow evidence," Archives of General Psychiatry, vol. 43, no. 2, pp. 114-124, 1986.

[21] M. J. Minzenberg, A. R. Laird, S. Thelen, C. S. Carter, and D. C. Glahn, "Meta-analysis of 41 functional neuroimaging studies of executive function in schizophrenia," Archives of General Psychiatry, vol. 66, no. 8, pp. 811-822, 2009.

[22] J. H. Callicott, A. Bertolino, V. S. Mattay et al., "Physiological dysfunction of the dorsolateral prefrontal cortex in schizophrenia revisited," Cerebral Cortex, vol. 10, no. 11, pp. 1078-1092, 2000.

[23] D. S. Manoach, D. Z. Press, V. Thangaraj et al., "Schizophrenic subjects activate dorsolateral prefrontal cortex during a working memory task, as measured by fMRI," Biological Psychiatry, vol. 45, no. 9, pp. 1128-1137, 1999.

[24] A. M. Achim and M. Lepage, "Episodic memory-related activation in schizophrenia: meta-analysis," British Journal of Psychiatry, vol. 187, pp. 500-509, 2005.

[25] D. M. Barch and A. Ceaser, "Cognition in schizophrenia: core psychological and neural mechanisms," Trends in Cognitive Sciences, vol. 16, no. 1, pp. 27-34, 2012.

[26] R. S. Kahn and R. S. Keefe, "Schizophrenia is a cognitive illness: time for a change in focus," JAMA Psychiatry, 2013.

[27] A. Meyer-Lindenberg, "From maps to mechanisms through neuroimaging of schizophrenia," Nature, vol. 468, no. 7321, pp. 194-202, 2010.

[28] A. Aleman, E. H. F. de Haan, S. A. Castner, G. V. Williams, and P. S. Goldman-Rakic, "Antipsychotics and working memory in Schizophrenia," Science, vol. 289, no. 5476, pp. 56-58, 2000.

[29] A. L. Mishara and T. E. Goldberg, "A meta-analysis and critical review of the effects of conventional neuroleptic treatment on cognition in schizophrenia: opening a closed book," Biological Psychiatry, vol. 55, no. 10, pp. 1013-1022, 2004.

[30] R. S. E. Keefe, J. A. Sweeney, H. Gu et al., "Effects of olanzapine, quetiapine, and risperidone on neurocognitive function in early psychosis: a randomized, double-blind 52-week comparison," The American Journal of Psychiatry, vol. 164, no. 7, pp. 1061-1071, 2007.

[31] E. J. Liemburg, H. Knegtering, H. C. Klein, R. Kortekaas, and A. Aleman, "Antipsychotic medication and prefrontal cortex activation: a review of neuroimaging findings," European Neuropsychopharmacology, vol. 22, no. 6, pp. 387-400, 2012.

[32] L. Krabbendam and A. Aleman, "Cognitive rehabilitation in schizophrenia: a quantitative analysis of controlled studies," Psychopharmacology, vol. 169, no. 3-4, pp. 376-382, 2003.

[33] T. Wykes, V. Huddy, C. Cellard, S. R. McGurk, and P. Czobor, "A meta-analysis of cognitive remediation for schizophrenia: methodology and effect sizes," The American Journal of Psychiatry, vol. 168, no. 5, pp. 472-485, 2011.

[34] S. M. Eack, G. E. Hogarty, R. Y. Cho et al., "Neuroprotective effects of cognitive enhancement therapy against gray matter loss in early schizophrenia: results from a 2-year randomized controlled trial," Archives of General Psychiatry, vol. 67, no. 7, pp. 674-682, 2010.

[35] A. Aleman and F. Laroi, Hallucinations: The Science of Idiosyncratic Perception, The American Psychological Association, Washington, DC, USA, 2008. 
[36] S. McCarthy-Jones, T. Trauer, A. Mackinnon, E. Sims, N. Thomas, and D. L. Copolov, "A new phenomenological survey of auditory hallucinations: evidence for subtypes and implications for theory and practice," Schizophrenia Bulletin, 2013.

[37] D. A. Silbersweig, E. Stern, C. Frith et al., "A functional neuroanatomy of hallucinations in schizophrenia," Nature, vol. 378, no. 6553, pp. 176-179, 1995.

[38] S. S. Shergill, M. J. Brammer, S. C. R. Williams, R. M. Murray, and P. K. McGuire, "Mapping auditory hallucinations in schizophrenia using functional magnetic resonance imaging," Archives of General Psychiatry, vol. 57, no. 11, pp. 1033-1038, 2000.

[39] B. R. Lennox, S. B. G. Park, I. Medley, P. G. Morris, and P. B. Jones, "The functional anatomy of auditory hallucinations in schizophrenia," Psychiatry Research, vol. 100, no. 1, pp. 13-20, 2000.

[40] I. E. C. Sommer, K. M. J. Diederen, J.-D. Blom et al., "Auditory verbal hallucinations predominantly activate the right inferior frontal area," Brain, vol. 131, no. 12, pp. 3169-3177, 2008.

[41] R. Jardri, A. Pouchet, D. Pins, and P. Thomas, "Cortical activations during auditory verbal hallucinations in schizophrenia: a coordinate-based meta-analysis," The American Journal of Psychiatry, vol. 168, no. 1, pp. 73-81, 2011.

[42] S. Kühn and J. Gallinat, "Quantitative meta-analysis on state and trait aspects of auditory verbal hallucinations in schizophrenia," Schizophrenia Bulletin, vol. 38, no. 4, pp. 779-786, 2012.

[43] R. E. Hoffman, R. Gueorguieva, K. A. Hawkins et al., "Temporoparietal transcranial magnetic stimulation for auditory hallucinations: safety, efficacy and moderators in a fifty patient sample," Biological Psychiatry, vol. 58, no. 2, pp. 97-104, 2005.

[44] A. Aleman, I. E. Sommer, and R. S. Kahn, "Efficacy of slow repetitive transcranial magnetic stimulation in the treatment of resistant auditory hallucinations in schizophrenia: a metaanalysis," Journal of Clinical Psychiatry, vol. 68, no. 3, pp. 416421, 2007.

[45] A. Aleman, "Use of repetitive transcranial magnetic stimulation for treatment in psychiatry," Clinical Psychopharmacology and Neuroscience, vol. 11, no. 2, pp. 53-59, 2013.

[46] A. Vercammen, H. Knegtering, R. Bruggeman et al., "Effects of bilateral repetitive transcranial magnetic stimulation on treatment resistant auditory-verbal hallucinations in schizophrenia: a randomized controlled trial," Schizophrenia Research, vol. 114, no. 1-3, pp. 172-179, 2009.

[47] C. W. Slotema, A. Aleman, Z. J. Daskalakis, and I. E. Sommer, "Meta-analysis of repetitive transcranial magnetic stimulation in the treatment of auditory verbal hallucinations: update and effects after one month," Schizophrenia Research, vol. 142, no. 13, pp. 40-45, 2012.

[48] F. Waters, P. Allen, A. Aleman et al., "Auditory hallucinations in schizophrenia and nonschizophrenia populations: a review and integrated model of cognitive mechanisms," Schizophrenia Bulletin, vol. 38, no. 4, pp. 683-693, 2012.

[49] J. C. Badcock, F. A. V. Waters, M. T. Maybery, and P. T. Michie, "Auditory hallucinations: failure to inhibit irrelevant memories," Cognitive Neuropsychiatry, vol. 10, no. 2, pp. 125-136, 2005.

[50] F. A. V. Waters, J. C. Badcock, P. T. Michie, and M. T. Maybery, "Auditory hallucinations in schizophrenia: intrusive thoughts and forgotten memories," Cognitive Neuropsychiatry, vol. 11, no. 1, pp. 65-83, 2006.

[51] M. F. Soriano, J. F. Jiménez, P. Román, and M. T. Bajo, "Intentional inhibition in memory and hallucinations: directed forgetting and updating," Neuropsychology, vol. 23, no. 1, pp. 6170, 2009.

[52] R.-P. Behrendt, "Underconstrained perception: a theoretical approach to the nature and function of verbal hallucinations," Comprehensive Psychiatry, vol. 39, no. 4, pp. 236-248, 1998.

[53] S. Grossberg, "How hallucinations may arise from brain mechanisms of learning, attention, and volition," Journal of the International Neuropsychological Society, vol. 6, no. 5, pp. 583$592,2000$.

[54] A. Vercammen and A. Aleman, "Semantic expectations can induce false perceptions in hallucination-prone individuals," Schizophrenia Bulletin, vol. 36, no. 1, pp. 151-156, 2010.

[55] K. Daalman, S. Verkooijen, E. M. Derks, A. Aleman, and I. E. Sommer, "The influence of semantic top-down processing in auditory verbal hallucinations," Schizophrenia Research, vol.139, no. 1-3, pp. 82-86, 2012.

[56] A. S. David, "Why we need more debate on whether psychotic symptoms lie on a continuum with normality," Psychological Medicine, vol. 40, no. 12, pp. 1935-1942, 2010.

[57] A. Aleman, E. Formisano, H. Koppenhagen, P. Hagoort, E. H. F. de Haan, and R. S. Kahn, "The functional neuroanatomy of metrical stress evaluation of perceived and imagined spoken words," Cerebral Cortex, vol. 15, no. 2, pp. 221-228, 2005.

[58] A. Vercammen, H. Knegtering, J. A. denBoer, E. J. Liemburg, and A. Aleman, "Auditory hallucinations in schizophrenia are associated with reduced functional connectivity of the temporo-parietal area," Biological Psychiatry, vol. 67, no. 10, pp. 912918, 2010.

[59] B. Curcic-Blake, E. Liemburg, A. Vercammen et al., "When broca goes uninformed: reduced information flow to Broca's area in Schizophrenia patients with auditory hallucinations," Schizophrenia Bulletin, vol. 39, no. 5, pp. 1087-1095, 2013.

[60] M. K. Johnson, S. Hashtroudi, and D. Stephen Lindsay, "Source monitoring," Psychological Bulletin, vol. 114, no. 1, pp. 3-28, 1993.

[61] M. K. Johnson and C. L. Raye, "Reality monitoring," Psychological Review, vol. 88, no. 1, pp. 67-85, 1981.

[62] R. S. E. Keefe, “The neurobiology of disturbances of the self: autonoetic agnosia in schizophrenia," in Insight and Psychosis, X. F. Amador and A. David, Eds., Oxford University Press, New York, NY, USA, 1998.

[63] C. D. Frith and D. J. Done, "Towards a neuropsychology of schizophrenia," British Journal of Psychiatry, vol. 153, pp. 437443, 1988.

[64] R. P. Bentall, G. A. Baker, and S. Havers, "Reality monitoring and psychotic hallucinations," British Journal of Clinical Psychology, vol. 30, no. 3, pp. 213-222, 1991.

[65] R. S. E. Keefe, M. C. Arnold, U. J. Bayen, J. P. McEvoy, and W. H. Wilson, "Source-monitoring deficits for self-generated stimuli in schizophrenia: multinomial modeling of data from three sources," Schizophrenia Research, vol. 57, no. 1, pp. 51-67, 2002.

[66] F. Waters, T. Woodward, P. Allen, A. Aleman, and I. Sommer, "Self-recognition deficits in schizophrenia patients with auditory hallucinations: a meta-analysis of the literature," Schizophrenia Bulletin, vol. 38, no. 4, pp. 741-750, 2012.

[67] J. S. Simons, S. W. Davis, S. J. Gilbert, C. D. Frith, and P. W. Burgess, "Discriminating imagined from perceived information engages brain areas implicated in schizophrenia," NeuroImage, vol. 32, no. 2, pp. 696-703, 2006.

[68] P. Allen, E. Amaro, C. H. Y. Fu et al., "Neural correlates of the misattribution of speech in schizophrenia," British Journal of Psychiatry, vol. 190, pp. 162-169, 2007. 
[69] B. Elvevåg and T. E. Goldberg, "Cognitive impairment in schizophrenia is the core of the disorder," Critical Reviews in Neurobiology, vol. 14, no. 1, pp. 1-21, 2000.

[70] R. W. Heinrichs, "The primacy of cognition in schizophrenia," The American Psychologist, vol. 60, no. 3, pp. 229-242, 2005.

[71] A. Aleman and A. S. David, "How to fill a half-full glass: emotion and schizophrenia," The American Psychologist, vol. 61, no. 1, pp. 75-76, 2006.

[72] A. Aleman and R. S. Kahn, "Strange feelings: do amygdala abnormalities dysregulate the emotional brain in schizophrenia?" Progress in Neurobiology, vol. 77, no. 5, pp. 283-298, 2005.

[73] C. G. Kohler, J. B. Walker, E. A. Martin, K. M. Healey, and P. J. Moberg, "Facial emotion perception in schizophrenia: a metaanalytic review," Schizophrenia Bulletin, vol. 36, no. 5, pp. 1009$1019,2010$.

[74] M. Hoekert, R. S. Kahn, M. Pijnenborg, and A. Aleman, "Impaired recognition and expression of emotional prosody in schizophrenia: review and meta-analysis," Schizophrenia Research, vol. 96, no. 1-3, pp. 135-145, 2007.

[75] F. Irani, S. Seligman, V. Kamath, C. Kohler, and R. C. Gur, "A meta-analysis of emotion perception and functional outcomes in schizophrenia," Schizophrenia Research, vol. 137, no. 1-3, pp. 203-211, 2012.

[76] H. Li, R. C. K. Chan, G. M. McAlonan, and Q.-Y. Gong, "Facial emotion processing in schizophrenia: a meta-analysis of functional neuroimaging data," Schizophrenia Bulletin, vol. 36, no. 5, pp. 1029-1039, 2010.

[77] A. Anticevic, J. X. Van Snellenberg, R. E. Cohen, G. Repovs, E. C. Dowd, and D. M. Barch, "Amygdala recruitment in schizophrenia in response to aversive emotional material: a metaanalysis of neuroimaging studies," Schizophrenia Bulletin, vol. 38, no. 3, pp. 608-621, 2012.

[78] D. Baas, A. Aleman, M. Vink, N. F. Ramsey, E. H. F. de Haan, and R. S. Kahn, "Evidence of altered cortical and amygdala activation during social decision-making in schizophrenia," NeuroImage, vol. 40, no. 2, pp. 719-727, 2008.

[79] K. N. Ochsner, S. A. Bunge, J. J. Gross, and J. D. E. Gabrieli, "Rethinking feelings: an fMRI study of the cognitive regulation of emotion," Journal of Cognitive Neuroscience, vol. 14, no. 8, pp. 1215-1229, 2002.

[80] K. McRae, B. Ciesielski, and J. J. Gross, "Unpacking cognitive reappraisal: goals, tactics, and outcomes," Emotion, vol. 12, no. 2, pp. 250-255, 2012.

[81] J. J. Gross, "Antecedent- and response-focused emotion regulation: divergent consequences for experience, expression, and physiology," Journal of Personality and Social Psychology, vol. 74, no. 1, pp. 224-237, 1998.

[82] A. Bechara, H. Damasio, A. R. Damasio, and G. P. Lee, "Different contributions of the human amygdala and ventromedial prefrontal cortex to decision-making," Journal of Neuroscience, vol. 19, no. 13, pp. 5473-5481, 1999.

[83] J. T. Buhle, J. A. Silvers, T. D. Wager et al., "Cognitive reappraisal of emotion: a meta-analysis of human neuroimaging studies," Cereb Cortex, 2013.

[84] D. Kimhy, J. Vakhrusheva, L. Jobson-Ahmed, N. Tarrier, D. Malaspina, and J. J. Gross, "Emotion awareness and regulation in individuals with schizophrenia: implications for social functioning," Psychiatry Research, vol. 200, no. 2-3, pp. 193-201, 2012.

[85] L. van der Meer, M. V. Wout, and A. Aleman, "Emotion regulation strategies in patients with schizophrenia," Psychiatry Research, vol. 170, no. 2-3, pp. 108-113, 2009.
[86] R. W. Morris, A. Sparks, P. B. Mitchell, C. S. Weickert, and M. J. Green, "Lack of cortico-limbic coupling in bipolar disorder and schizophrenia during emotion regulation," Translational Psychiatry, vol. 2, article e90, 2012.

[87] R. M. Seyfarth and D. L. Cheney, "Affiliation, empathy, and the origins of theory of mind," Proceedings of the National Academy of Sciences of the USA, vol. 110, supplement 2, pp. 10349-10356, 2013.

[88] L. Harrington, R. Langdon, R. J. Siegert, and J. McClure, "Schizophrenia, theory of mind, and persecutory delusions," Cognitive Neuropsychiatry, vol. 10, no. 2, pp. 87-104, 2005.

[89] R. Corcoran, G. Mercer, and C. D. Frith, "Schizophrenia, symptomatology and social inference: investigating "theory of mind" in people with schizophrenia," Schizophrenia Research, vol. 17, no. 1, pp. 5-13, 1995.

[90] R. Saxe and N. Kanwisher, "People thinking about thinking people: the role of the temporo-parietal junction in 'theory of mind"' NeuroImage, vol. 19, no. 4, pp. 1835-1842, 2003.

[91] D. Samson, I. A. Apperly, C. Chiavarino, and G. W. Humphreys, "Left temporoparietal junction is necessary for representing someone else's belief," Nature Neuroscience, vol. 7, no. 5, pp. 499500, 2004.

[92] P. Ruby and J. Decety, "What you believe versus what you think they believe: a neuroimaging study of conceptual perspectivetaking," European Journal of Neuroscience, vol. 17, no. 11, pp. 2475-2480, 2003.

[93] A. D. Rowe, P. R. Bullock, C. E. Polkey, and R. G. Morris, “"Theory of mind' impairments and their relationship to executive functioning following frontal lobe excisions," Brain, vol. 124, pp. 600-616, 2001.

[94] D. Samson, I. A. Apperly, U. Kathirgamanathan, and G. W. Humphreys, "Seeing it my way: a case of a selective deficit in inhibiting self-perspective," Brain, vol. 128, no. 5, pp. 1102-1111, 2005.

[95] H. L. Gallagher and C. D. Frith, "Functional imaging of 'theory of mind"' Trends in Cognitive Sciences, vol. 7, no. 2, pp. 77-83, 2003.

[96] K. Vogeley, P. Bussfeld, A. Newen et al., "Mind reading: neural mechanisms of theory of mind and self-perspective," NeuroImage, vol. 14, no. 1, pp. 170-181, 2001.

[97] A. R. Aron, T. W. Robbins, and R. A. Poldrack, "Inhibition and the right inferior frontal cortex," Trends in Cognitive Sciences, vol. 8, no. 4, pp. 170-177, 2004.

[98] S. G. Shamay-Tsoory, J. Aharon-Peretz, and Y. Levkovitz, "The neuroanatomical basis of affective mentalizing in schizophrenia: comparison of patients with schizophrenia and patients with localized prefrontal lesions," Schizophrenia Research, vol. 90, no. 1-3, pp. 274-283, 2007.

[99] S. G. Shamay-Tsoory and J. Aharon-Peretz, "Dissociable prefrontal networks for cognitive and affective theory of mind: a lesion study," Neuropsychologia, vol. 45, no. 13, pp. 3054-3067, 2007.

[100] G. Modinos, R. Renken, S. G. Shamay-Tsoory, J. Ormel, and A. Aleman, "Neurobiological correlates of theory of mind in psychosis proneness," Neuropsychologia, vol. 48, no. 13, pp. 37153724, 2010.

[101] L. van der Meer, N. A. Groenewold, W. A. Nolen, M. Pijnenborg, and A. Aleman, "Inhibit yourself and understand the other: neural basis of distinct processes underlying Theory of Mind," NeuroImage, vol. 56, no. 4, pp. 2364-2374, 2011. 
[102] L. van der Meer, N. A. Groenewold, M. Pijnenborg, and A. Aleman, "Psychosis-proneness and neural correlates of selfinhibition in theory of mind," PLOS ONE, vol. 8, no. 7, Article ID e67774, 2013.

[103] B. Derntl, A. Finkelmeyer, T. K. Toygar et al., "Generalized deficit in all core components of empathy in schizophrenia," Schizophrenia Research, vol. 108, no. 1-3, pp. 197-206, 2009.

[104] D. Baas, M. Van't Wout, A. Aleman, and R. S. Kahn, "Social judgement in clinically stable patients with schizophrenia and healthy relatives: behavioural evidence of social brain dysfunction," Psychological Medicine, vol. 38, no. 5, pp. 747-754, 2008.

[105] T. Jellema, J. Lorteije, S. van Rijn et al., "Involuntary interpretation of social cues is compromised in autism spectrum disorders," Autism Research, vol. 2, no. 4, pp. 192-204, 2009.

[106] M. van 't Wout, S. van Rijn, T. Jellema, R. S. Kahn, and A. Aleman, "Deficits in implicit attention to social signals in schizophrenia and high risk groups: behavioural evidence from a new illusion," PLoS ONE, vol. 4, no. 5, Article ID e5581, 2009.

[107] X. F. Amador and A. S. David, Eds., Insight and Psychosis: awareness of Illness in Schizophrenia and Related Disorders, Oxford University Press, Oxford, UK, 2nd edition, 2004.

[108] A. S. David, "Insight and psychosis," British Journal of Psychiatry, vol. 156, pp. 798-808, 1990.

[109] A. S. David, “The clinical importance of insight: an overview," in Insight and Psychosis: Awareness of Illness in Schizophrenia and Related Disorders, X. F. Amador and A. S. David, Eds., Oxford University Press, Oxford, UK, 2nd edition, 2004.

[110] A. Aleman, N. Agrawal, K. D. Morgan, and A. S. David, "Insight in psychosis and neuropsychological function: meta-analysis," British Journal of Psychiatry, vol. 189, pp. 204-212, 2006.

[111] A. Nair, E. C. Palmer, A. Aleman, and A. S. David, "Relationship between cognitive functioning and clinical and cognitive insight in psychotic disorders: a review and meta-analysis," Schizophrenia Research, vol. 152, no. 1, pp. 191-200, 2014.

[112] P. J. Quee, L. van der Meer, R. Bruggeman et al., "Insight in psychosis: relationship with neurocognition, social cognition and clinical symptoms depends on phase of illness," Schizophrenia Bulletin, vol. 37, no. 1, pp. 29-37, 2011.

[113] S. Epstein, "Integration of the cognitive and the psychodynamic unconscious," The American Psychologist, vol. 49, no. 8, pp. 709724, 1994.

[114] T. D. Wilson, S. Lindsey, and T. Y. Schooler, "A model of dual attitudes," Psychological Review, vol. 107, no. 1, pp. 101-126, 2000.

[115] S. L. Koole, A. Dijksterhuis, and A. van Knippenberg, "What's in a name: implicit self-esteem and the automatic self," Journal of Personality and Social Psychology, vol. 80, no. 4, pp. 669-685, 2001.

[116] S. C. Johnson, L. C. Baxter, L. S. Wilder, J. G. Pipe, J. E. Heiserman, and G. P. Prigatano, "Neural correlates of self-reflection," Brain, vol. 125, no. 8, pp. 1808-1814, 2002.

[117] D. T. Stuss, G. G. Gallup Jr., and M. P. Alexander, “The frontal lobes are necessary for 'theory of mind"' Brain, vol. 124, no. 2, pp. 279-286, 2001.

[118] L. van der Meer, S. Costafreda, A. Aleman, and A. S. David, "Self-reflection and the brain: a theoretical review and meta-analysis of neuroimaging studies with implications for schizophrenia," Neuroscience and Biobehavioral Reviews, vol. 34, no. 6, pp. 935-946, 2010.

[119] R. Kemp and A. S. David, "Insight and compliance," in Treatment Compliance and the Therapeutic Alliance, pp. 61-84, Harwood Academic Publishers, Amsterdam, The the Netherlands, 1997.
[120] L. van der Meer, A. E. de Vos, A. P. Stiekema et al., "Insight in schizophrenia: involvement of self-reflection networks?" Schizophrenia Bulletin2013, vol. 39, no. 6, pp. 1288-1295.

[121] A. T. Beck, E. Baruch, J. M. Balter, R. A. Steer, and D. M. Warman, "A new instrument for measuring insight: the beck cognitive insight scale," Schizophrenia Research, vol. 68, no. 2-3, pp. 319-329, 2004.

[122] R. J. Davidson, "Affective style, psychopathology, and resilience: brain mechanisms and plasticity," The American Psychologist, vol. 55, no. 11, pp. 1196-1214, 2000.

[123] G. H. Pijnenborg, J. M. Spikman, B. F. Jeronimus, and A. Aleman, "Insight in schizophrenia: associations with empathy," European Archives of Psychiatry and Clinical Neurosciences, vol. 263, no. 4, pp. 299-307, 2013.

[124] H. G. Engen and T. Singer, "Empathy circuits," Current Opinion in Neurobiology, vol. 23, no. 2, pp. 275-282, 2013.

[125] G. Foussias and G. Remington, "Negative symptoms in schizophrenia: avolition and occam's razor," Schizophrenia Bulletin, vol. 36, no. 2, pp. 359-369, 2010.

[126] M. Kiang, B. K. Christensen, G. Remington, and S. Kapur, "Apathy in schizophrenia: clinical correlates and association with functional outcome," Schizophrenia Research, vol. 63, no. 1-2, pp. 79-88, 2003.

[127] R. Bottlender, A. Strauss, and H.-J. Möller, "Social disability in schizophrenic, schizoaffective and affective disorders 15 years after first admission," Schizophrenia Research, vol. 116, no. 1, pp. 9-15, 2010.

[128] R. van Reekum, D. T. Stuss, and L. Ostrander, "Apathy: why care?" Journal of Neuropsychiatry and Clinical Neurosciences, vol. 17, no. 1, pp. 7-19, 2005.

[129] D. T. Stuss, R. van Reekum, and K. J. Murphy, "Differentiation of states and causes of apathy," in The Neuropsychology of Emotion, J. Borod, Ed., Oxford University Press, New York, NY, USA, 2000.

[130] R. Levy and B. Dubois, "Apathy and the functional anatomy of the prefrontal cortex-basal ganglia circuits," Cerebral Cortex, vol. 16, no. 7, pp. 916-928, 2006.

[131] E. Liemburg, S. Castelein, R. Stewart et al., "Two subdomains of negative symptoms in psychotic disorders: established and confirmed in two large cohorts," Journal of Psychiatric Research, vol. 47, no. 6, pp. 718-725, 2013.

[132] R. M. Roth, L. A. Flashman, A. J. Saykin, T. W. McAllister, and R. Vidaver, "Apathy in schizophrenia: reduced frontal lobe volume and neuropsychological deficits," The American Journal of Psychiatry, vol. 161, no. 1, pp. 157-159, 2004.

[133] J. H. Callicott, A. Bertolino, M. F. Egan, V. S. Mattay, F. J. P. Langheim, and D. R. Weinberger, "Selective relationship between prefrontal $\mathrm{N}$-acetylaspartate measures and negative symptoms in schizophrenia," The American Journal of Psychiatry, vol. 157, no. 10, pp. 1646-1651, 2000.

[134] E. C. Dowd and D. M. Barch, "Anhedonia and emotional experience in schizophrenia: neural and behavioral indicators," Biological Psychiatry, vol. 67, no. 10, pp. 902-911, 2010.

[135] J. J. Simon, A. Biller, S. Walther et al., "Neural correlates of reward processing in schizophrenia: relationship to apathy and depression," Schizophrenia Research, vol. 118, no. 1-3, pp. 154161, 2010.

[136] G. E. Tsai and P.-Y. Lin, "Strategies to enhance N-Methyl-DAspartate receptor-mediated neurotransmission in schizophrenia, a critical review and meta-analysis," Current Pharmaceutical Design, vol. 16, no. 5, pp. 522-537, 2010. 
[137] M. J. Noetzel, C. K. Jones, and P. J. Conn, "Emerging approaches for treatment of schizophrenia: modulation of glutamatergic signaling," Discovery Medicine, vol. 14, no. 78, pp. 335-343, 2012.

[138] J. J. Dlabač-de Lange, R. Knegtering, and A. Aleman, "Repetitive transcranial magnetic stimulation for negative symptoms of schizophrenia: review and meta-analysis," Journal of Clinical Psychiatry, vol. 71, no. 4, pp. 411-418, 2010.

[139] R. Prikryl and H. P. Kucerova, "Can repetitive transcranial magnetic stimulation be considered effective treatment option for negative symptoms of schizophrenia?" The Journal of ECT, vol. 29, no. 1, pp. 67-74, 2013.

[140] G. P. Strauss, W. R. Keller, R. W. Buchanan et al., "Nextgeneration negative symptom assessment for clinical trials: validation of the Brief Negative Symptom Scale," Schizophrenia Research, vol. 142, no. 1-3, pp. 88-92, 2012.

[141] P. Allen, F. Larøi, P. K. McGuire, and A. Aleman, "The hallucinating brain: a review of structural and functional neuroimaging studies of hallucinations," Neuroscience and Biobehavioral Reviews, vol. 32, no. 1, pp. 175-191, 2008.

[142] A. Vercammen, H. Knegtering, E. J. Liemburg, J. A. D. Boer, and A. Aleman, "Functional connectivity of the temporo-parietal region in schizophrenia: effects of rTMS treatment of auditory hallucinations," Journal of Psychiatric Research, vol. 44, no. 11, pp. 725-731, 2010. 

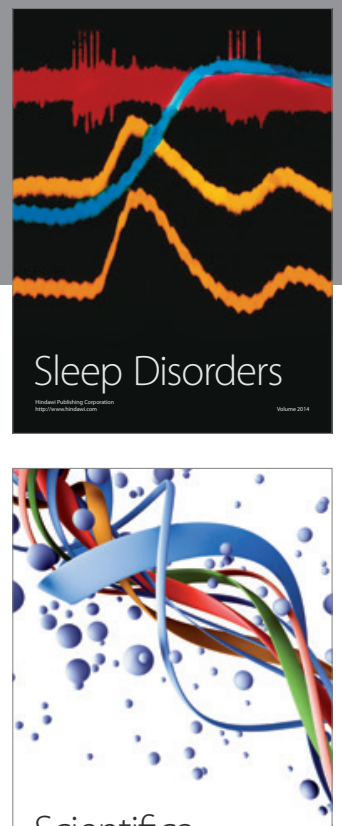

Scientifica
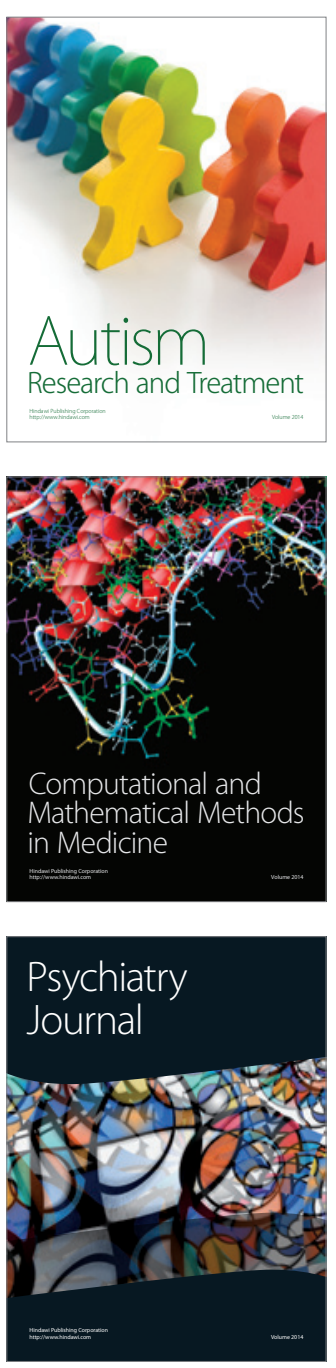
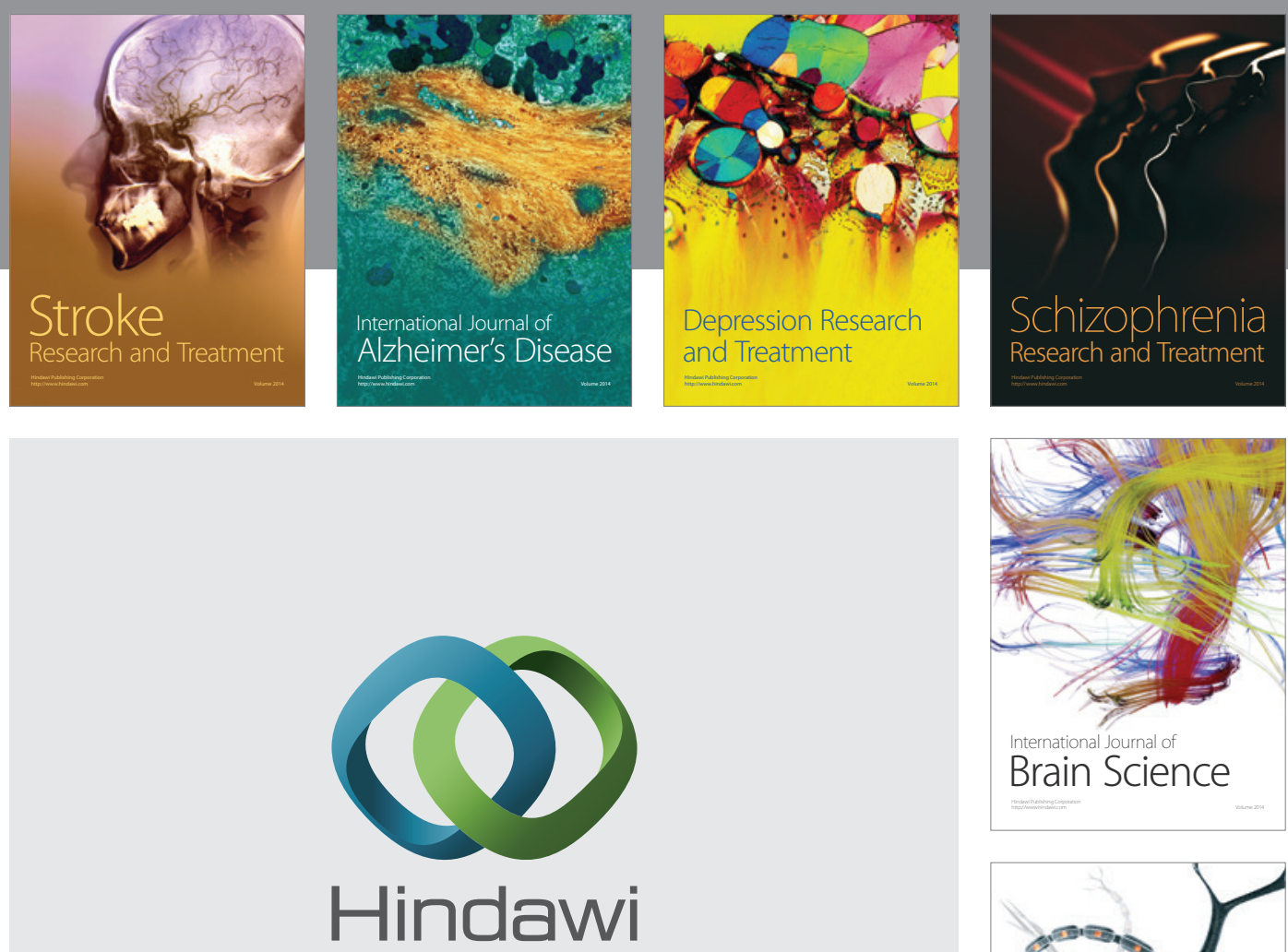

Submit your manuscripts at

http://www.hindawi.com
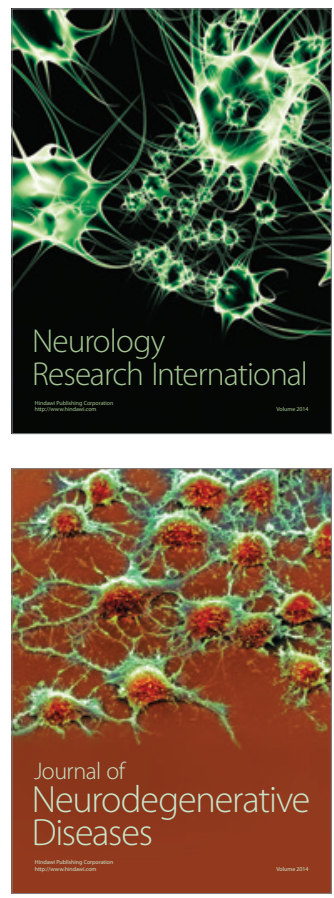

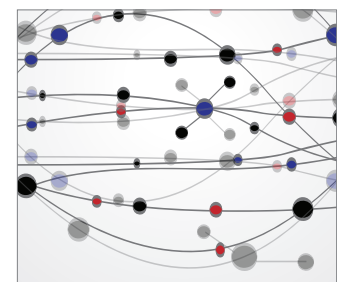

The Scientific World Journal
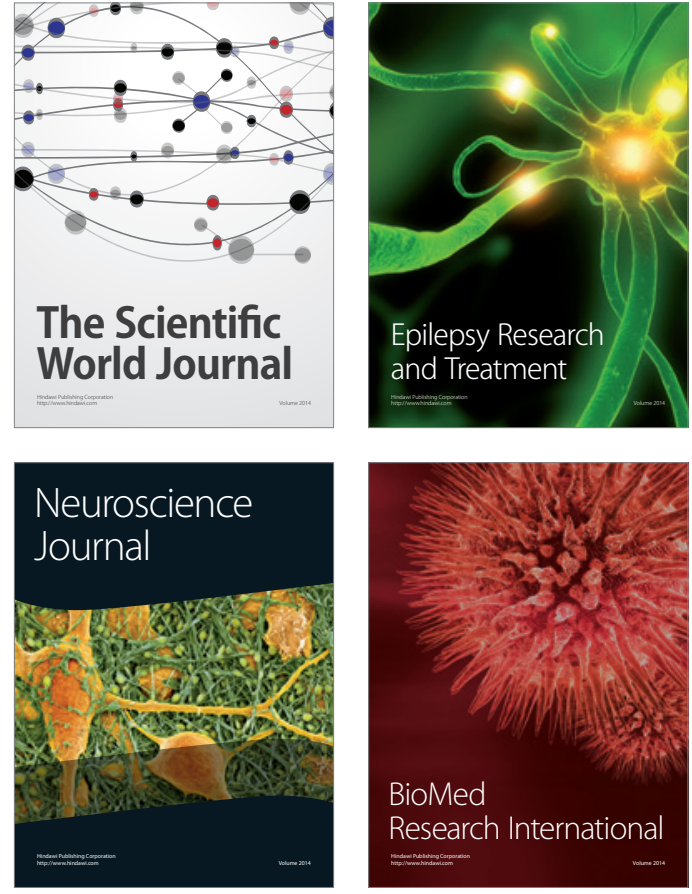

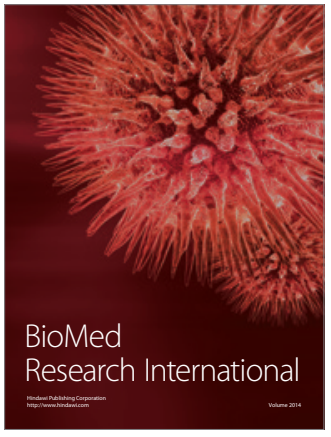

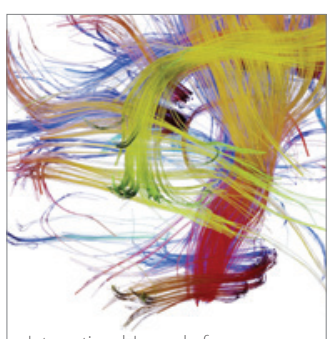

Brain Science

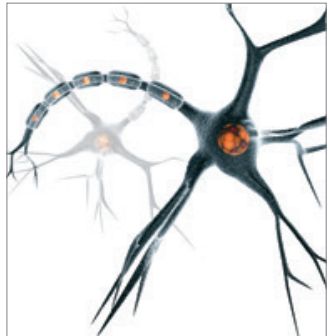

Neural Plasticity
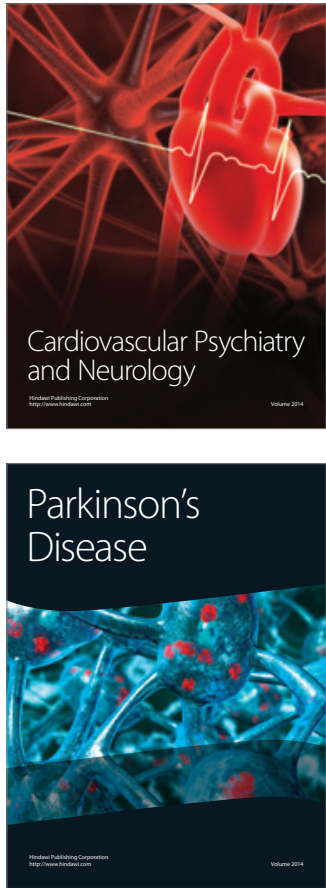